\title{
Antimicrobial Activity of MTA Fillapex, Real Seal SE, Acroseal and Zinc oxide Eugenol Sealers against Enterococcus Faecalis and Candida Albicans
}

\author{
Hena Rahman ${ }^{1}$, Ramesh Chandra ${ }^{2}$, Divya Chowdhary ${ }^{3}$, Shailja Singh ${ }^{4}$, \\ Supratim Tripathi ${ }^{4}$, Syed Zaki Anwar \\ ${ }^{1}$ Assistant Professor, Dentistry Department, Rajkiya Medical College, Jalaun. \\ ${ }^{2}$ Professor, Department Of Conservative Dentistry And Endodontics, Career Pg Institute Of Dental Sciences \\ And Hospital. \\ ${ }^{3}$ Pg Student, Department Of Conservative Dentistry And Endodontics, Career Pg Institute Of Dental Sciences \\ And Hospital. \\ ${ }^{4}$ Reader, Department Of Conservative Dentistry And Endodontics, Career Pg Institute Of Dental Sciences And \\ Hospital. \\ "Corresponding Author
}

\begin{abstract}
:
Aim:The aim of the present study was to evaluate the antimicrobial activity of different root canal sealers (MTA Fillapex, Real Seal SE, Acroseal and Zinc Oxide Eugenol) against Enterococcus fecalis and Candida albicans. Materials and method: The antimicrobial effect of different root canal sealers was evaluated by the direct contact with agar diffusion method. The freshly prepared mixed sealers were placed in prepared wells of agar plates inoculated with E. faecalis and C.albicans separately. All plates were incubated for 7 days at $37^{\circ} \mathrm{C}$ under aerobic conditions, and zones of inhibition were measured at 24, 48, 72 hours and 7 days. All statistical analyses were performed with the SPSS (PC version 10 software, IBM, NY, USA) statistical software.

Result : The sealer MTA fillapex showed highest antimicrobial activity against C. albicans $(16.0 \pm 0.20 \mathrm{~mm})$ followed by Real Seal SE $(15.2 \pm 0.20 \mathrm{~mm})$. Amongst the tested sealers only MTA Fillapex showed antimicrobial activity against E. faecalis $(12.40 \pm 0.20 \mathrm{~mm})$. No antimicrobial activity was observed with zinc oxide eugenol and Acroseal.

Conclusion : Amongst the tested sealers MTA Fillapex and Real Seal SE both showed antimicrobial activity against C.albicans whereas only MTA Fillapex showed antibacterial activity against E. faecalis. Acroseal and Zinc Oxide Eugenol did not show any antimicrobial activity.
\end{abstract}

Keywords: Agar Diffusion Test, E.faecalis, C.albicans, Root canal sealers

\section{Introduction}

The main purpose of endodontic therapy is to provide an inert atmosphere and to prevent microbes from infecting or re-infecting the root canal or the periapical tissues. [1] Antimicrobial activity has been long talked about and in present scenario, a major part is played by the property and the capacity of the sealer to do the same. Hence a good root canal sealer with significant antibacterial properties would be beneficial for further reduction of residual microorganism. Candida albicans and Enterococcus faecalis are the two microbes that have been the major reason for the failure of endodontic treatment. Maximum number of retreatment cases are reported because of these two. [2,3]

The success of obturation depends upon elimination of microorganisms through mechanical cleaning and shaping, supplemented by antibacterial irrigants, adequate filling of the empty space, and use of antimicrobial dressings between appointments, if necessary. [4,5] However, these procedures do not result in complete sterility of the root canal space. [6] One of the ideal requirement of root canal sealer is antimicrobial activity. Therefore, antimicrobial agents are added to root canal sealers to improve their antibacterial effect. $[7,8]$

MTA fillapex (Angelus Solucxoes Odontologicas, Londrina, Brazil) is mineral trioxide aggregate (MTA) based sealer, it consists of resins, silica, and MTA. It has high radioopacity, low solubility, low expansion during setting, promotes deposition of hard tissue and known for its antibacterial effect. [9]

Real Seal SE (SybronEndo, Orange, CA) is fourth generation methacrylate resin based sealer, in that they have further eliminated the separate etching/ bonding step. [10]

Acroseal (Septodont, St. Maurdes-Fosses Cedex, France) is calcium hydroxide-based sealer, it appears to have lower solubility than other calcium hydroxide sealers, probably because of its epoxy resin component. [11] 
The agar diffusion test (ADT) has been widely used to test the antimicrobial activity of root canal sealers. This method allows direct comparisons between materials and also indicates which sealers are more likely to have antimicrobial activity within the root canal system. Besides that, its results are highly influenced by the diffusibility of the material across the medium. [6,12,13] Therefore, aim of the present study is to evaluate the antimicrobial activity of different root canal sealers against E.faecalis and C.albicans using agar diffusion method.

\section{Materials And Method}

The materials evaluated for antimicrobial activity were MTA Fillapex, Real Seal SE, Acroseal, Zinc Oxide Eugenol cement (SS White, Rio de Janeiro, RJ, Brazil) against E. faecalis (ATCC 24212) and C. albicans (ATCC 24433) strains.

The bacterial strains were cultivated in a brain heart infusion broth (Difco; BD Diagnostic Systems, Sparks, MD, USA). The fungal strains were cultivated in Sabouraud's dextrose agar broth (Difco; BD Diagnostic Systems) in 48 hours. The broth culture suspensions were prepared and adjusted to No. 1 McFarland standard (approximately $3 \times 108$ cells $/ \mathrm{mL}$ ). Aliquots of the suspension containing E. faecalis and C. albicans were spread on four different 140-mm diameter Petri dishes containing Mueller-Hinton Agar medium (Merck, Darmstadt, Germany) for each microbial strain. Excess inoculum was removed with a pipette and the inoculated plates were dried for 15 minutes at $37^{\circ} \mathrm{C}$. Each plate was divided evenly into 4 sections. In each section of each plate, a well $4 \mathrm{~mm}$ in diameter was created with a sterile stainless steel cylinder. All the tested sealers were mixed according to the manufacturer's instructions. A sample of each freshly mixed dental material was placed into wells in each section of the plates.

All plates were incubated for 7 days at $37^{\circ} \mathrm{C}$ under aerobic conditions, and zones of growth inhibition were measured at 24, 48, 72 hours and 7 days using a plastic ruler and was recorded for each material. The results thus obtained were statistically analyzed using the two-way analysis of vari $a$ ance (ANOVA). The effects of each factor were analyzed by one-way ANOVA, and the inhibition zones associated with each sealer and each duration of incubaᄀtion were compared by Tukey's post hoc test. The value of $\mathrm{p}<0.5$ is considered significant. All statistical analyses were performed with the SPSS (PC version 10 software, IBM, NY, USA) statistical software package.

\section{Results}

The present study comparatively evaluate the antimicrobial activity of different endodontic sealers against E. Fecalis and C. Albicans. Total 16 samples for each species were taken and randomized equally in four groups (4 samples/group) and treated with MTA-Fillapex (MTA), Real seal SE (RS), Acroseal (AC) and Zinc oxide eugenol (ZOE). The outcome measure of the study was zone of inhibition (mm) assessed at 24, 48, $72 \mathrm{hrs}$ and 7 days post treatment.

\section{Candida Albicans}

The post treatment C. Albicans zone of inhibition of four groups (MTA, RS, AC and ZOE) is summarized in Table 1. After $24 \mathrm{hrs,} \mathrm{the} \mathrm{C.} \mathrm{Albicans} \mathrm{mean} \mathrm{zone} \mathrm{of} \mathrm{inhibition} \mathrm{was} \mathrm{highest} \mathrm{in} \mathrm{MTA} \mathrm{(21.0 \pm 0.15}$ $\mathrm{mm}$ ) followed by RS $(17.2 \pm 0.20 \mathrm{~mm})$. Further, in these groups, it decrease gradually after 24 hrs till 7 th day and the decrease was evident similar among the groups. However, in ZOE and AC, it was found absent at all periods, thus not comparable with other groups (Fig 1).

\section{Enterococcus Faecalis}

The post treatment E. Faecalis zone of inhibition of four groups (MTA, RS, AC and ZOE) is summarized in Table 2. After $24 \mathrm{hrs,} \mathrm{the} \mathrm{E.faecalis} \mathrm{mean} \mathrm{zone} \mathrm{of} \mathrm{inhibition} \mathrm{was} \mathrm{found} \mathrm{maximum} \mathrm{around} \mathrm{MTA}$ $(16.40 \pm 0.15 \mathrm{~mm})$ and decreases till 7 th day $(12.40 \pm 0.20 \mathrm{~mm})$. No other group showed any antimicrobial activity against E.faecalis at any time interval (Fig 1).

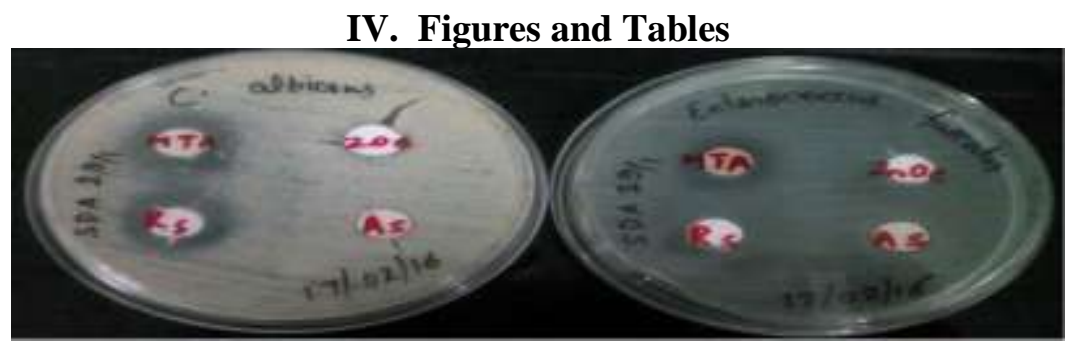

zig 1 showing zones of inhibition against C.albicans and E.faecalis 
Table 1: Post treatment C. Albicans zone of inhibition (Mean $\pm S D, n=4$ ) of four groups (in $m m$ ) and their effect on fungal strains.

\begin{tabular}{|l|l|l|l|l|l|}
\hline Periods & 24 hrs & 48hr & 72hr & 7day & Effect \\
\hline MTA & $21.0 \pm 0.15$ & $19.0 \pm 0.15$ & $18.0 \pm 0.15$ & $16.0 \pm 0.20$ & Sensitive \\
\hline RS & $17.2 \pm 0.20$ & $16.1 \pm 0.20$ & $15.2 \pm 0.20$ & $15.2 \pm 0.20$ & Sensitive \\
\hline AC & $00 \pm 0.00$ & $0.00 \pm 0.00$ & $0.00 \pm 0.00$ & $0.00 \pm 0.00$ & Resistant \\
\hline ZOE & $0.00 \pm 0.00$ & $0.00 \pm 0.00$ & $0.00 \pm 0.00$ & $0.00 \pm 0.00$ & Resistant \\
\hline
\end{tabular}

Table 2: Post treatment E. faecalis zone of inhibition (Mean $\pm \mathrm{SD}, \mathrm{n}=4$ ) of four groups (in $\mathrm{mm}$ ) and their effect on bacterial strains.

\begin{tabular}{|l|l|l|l|l|l|}
\hline Group & $\mathbf{2 4 h r}$ & $\mathbf{4 8 h r}$ & $\mathbf{7 2 h r}$ & 7 Day & Effect \\
\hline Mta & $16.40 \pm 0.15$ & $15.40 \pm 0.20$ & $14.20 \pm 0.15$ & $12.40 \pm 0.20$ & Sensitive \\
\hline Rs & $0.00 \pm 0.00$ & $0.00 \pm 0.00$ & $0.00 \pm 0.00$ & $0.00 \pm 0.00$ & Resistant \\
\hline Ac & $0.00 \pm 0.00$ & $0.00 \pm 0.00$ & $0.00 \pm 0.00$ & $0.00 \pm 0.00$ & Resistant \\
\hline Zoe & $0.00 \pm 0.00$ & $0.00 \pm 0.00$ & $0.00 \pm 0.00$ & $0.00 \pm 0.00$ & Resistant \\
\hline
\end{tabular}

\section{Discussion}

The selected microbes for this study was arbitrary to represent bacteria and fungus that have been commonly isolated from necrotic canals. E.faecalis is one of the most commonly isolated microorganisms from refractory periapical periodontitis[14] and it is one of the most drug resistant bacteria and has ability to survive up to 12 months even under nutrient deprived conditions in the root canal after routine root canal therapy. $[15,16]$

C.albicans is more often isolated from infected root canals, and is one of the common microorganisms that survive chemo-mechanical procedures and the application of root canal medicaments. [17]

In this study, the agar diffusion test was used, which is the most widely used in vitro method for the evaluation of antimicrobial activity. [18] This method allows direct comparisons between materials and also indicates which sealers are more likely to have antimicrobial activity within the root canal system. [19] Besides that, its results are highly influenced by the diffusibility of the material across the medium. [4] Most endodontic sealers possess antimicrobial components that need to be released from the sealer matrix to be effective. [19]

MTA Fillapex is resin salicylate and calcium silicate based root canal sealer. In the present study, MTA Fillapex was found to be most effective at $24 \mathrm{hr}$ against C.albicans and E.faecalis, after that its antimicrobial activity decreases at $48 \mathrm{hr}, 72 \mathrm{hr}$ and 7 th day respectively. On the contrary to our results Morgental et al stated that MTA Fillapex was effective before setting but not effective 7 days after setting. Such discrepancies are probably due to methodology. [9] The antimicrobial activity of MTA was reported by Torabinejad et al., who detected its efficiency against some facultative bacteria; however, no activity was found against E. faecalis, Staphylococcus aureus, Bacillus subtilis, and Escherichia coli or against anaerobic bacteria. [20] However, Stowe et al assessed the antimicrobial properties of MTA and found that it inhibited the growth of both E. faecalis and Streptococcus sanguis. [21] MTA contains calcium oxide, which forms calcium hydroxide on contact with water, which gives antibacterial property to MTA. [20,22,23] MTA Fillapex contains calcium silicate cement and with the moisture from dentin the hydration reactions of calcium silicates begins and calcium silicate hydrogel and calcium hydroxide exists which gives the high $\mathrm{PH}$ and antibacterial property to MTA Fillapex. [24] In present study antimicrobial property of MTA Fillapex could be related to calcium silicate, MTA and resin ingredient.

The results of present study showed that the most resistant microorganism to the antimicrobial activity of all sealers tested was Enterococcus faecalis except MTA Fillapex. This is one of the most resistant species usually found in root canal infection. The sealer MTA fillapex showed highest antimicrobial activity against C.albicans followed by Real Seal. No antimicrobial activity was observed with zinc oxide eugenol and Acroseal. In other study conducted by Filho et al showed different results from this study, where $\mathrm{ZnO}$ sealer demonstrated better antimicrobial activity than MTA-based cements. [25] The reason why ZnO sealer had better antimicrobial activity than MTA was not mentioned in the article. Eugenol content of $\mathrm{ZnO}$ sealer is generally known for its antimicrobial activity.

Although, Acroseal is a calcium hydroxide based sealer but it has shown no antimicrobial activity in this study, this result is in accordance with Tanomaru et al [26] but not with Pinheiro et al [27] where Acroseal was effective against E.faecalis.

Real Seal SE is a fourth generation resin based sealer which showed no antibacterial activity but little antifungal activity against C.albicans. This result is partially in accordance with Keshtkar and Riaz where Real Seal SE was ineffective against E.faecalis in ADT. [28] However, the results of present study was in vitro only, therefore more clinical trials are needed to confirm the effectiveness of tested sealers.

\section{Conclusion}


Amongst the tested sealers MTA Fillapex and Real Seal SE both showed antifungal activity whereas only MTA Fillapex was effective against E.faecalis, rest of the materials did not depict any antimicrobial activity in vitro.The introduction of the paper should explain the nature of the problem, previous work, purpose, and the contribution of the paper. The contents of each section may be provided to understand easily about the paper.

\section{References}

[1]. Love RM, McMillan MD, Jenkinson HF. Invasion of dentinal tubules by oral streptococci is associated with collagen recognition mediated by the antigen I/II family of polypeptides. Infect Immun 1997;65:5157-64.

[2]. Nair PN, Sjogren U, Krey G, Kahnberg KE, Sundqvist G. Intraradicular bacteria and fungi in root-filled, asymptomatic human teeth with therapy resistant periapical lesions: A long term light and electron microscopic follow up study. J Endod 1990;16:580-8.

[3]. Baumgartner JC, Watts CM, Xia T. Occurance of Candida albicans in infections of endodontic origin. J Endod 2000;26:695-8

[4]. Sundqvist G, Figdor D, Persson S, Sjogren U. Microbiologi analysis of teeth with failed endodontic treatment and the outcome of conservative re-treatment. Oral Surg Oral Med. Oral Pathol Oral Radiol Endod. 1998;85:86-93.

[5]. Reit C, Dahlen G. Decision making analysis of endodontic treatment strategies in teeth with apical periodontitis. Int Endod J 1988; 21(5):291-9.

[6]. Abdulkader A, Duguid R, Saunders EM. The antimicrobial activity of endoาdontic sealers to anaerobic bacteria. Int Endod J 1996; 29(4):280-3

[7]. Al-Khatib ZZ, Baum RH, Morse DR, Yesilsoy C, Bhambhani S, Furst ML. The antimicrobial effect of various enododontic sealers. Oral Surg Oral Med Oral Pathol 1990; 70(6):784-90

[8]. Grossman L. Antimicrobial effect of root canal cements. J Endod 1980; 6(6):594-7.

[9]. Morgental RD, Vier-Pelisser FV, Oliveira SD, Antunes FC, Cogo DM, Kopper PM. Antibacterial activity of two MTA-based root canal sealers. Int Endod J 2011;44:1128-33.

[10]. Radovic I, Monticelli F, Goracci C, Vulicevic ZR, Ferrari M. Self-adhesive resin cements: a literature review. J Adhes Dent 2008;10:251-8.

[11]. A. U. Eldeniz, K. Mustafa, D. Ørstavik, J. E. Dahl. Cytotoxicity of new resin-, calcium hydroxide- and silicone-based root canal sealers on fibroblasts derived from human gingiva and L929 cell lines. International Endodontic Journal 2007;40(5) : 329-37.

[12]. Mickel AK, Nguyen TH, Chogle S. Antimicrobial activity of endodontic sealers on Enterococcus faecalis. J Endod 2003; 29(4):257-8

[13]. Lai CC, Huang FM, Yang HW, Chan Y, Huang MS, Chou MY, and other. Antimicrobial activity of four root canal sealers against endodontic pathongens. Clin Oral Investig 2001; 5(4):236-9.

[14]. Stuart CH, Schwartz SA, Beeson TJ, Owatz CB. Enterococcus faecalis: Its role in root canal treatment failure and current concepts in retreatment. J Endod 2006;32:93-8.

[15]. Sedgley CM, Lennan SL, Appelbe OK. Survival of Enterococcus faecalis in root canals ex vivo. Int Endod J 2005;38:735-42.

[16]. Portenier I, Haapasalo H, Orstavik D, Yamauchi M, Haapasalo M. Inactivation of the antibacterial activity of iodine potassium iodide and chlorhexidine digluconate against Enterococcus faecalis by dentin, dentin matrix, type-I collagen, and heat-killed microbial whole cells. J Endod 2002;28:634-7.

[17]. Balakrishnan R, Dhole TK, Dubey S, Boruah LC, Langde SP. Comparative antimicrobial and antifungal efficacy of BioPure MTAD, Metapex and aztreonam on Enterococcus faecalis and Candida albicans. ENDO 2013;7:47-51.

[18]. Miyagak Daniela Cristina et.al. In vitro evaluation of the antimicrobial activity of endodontic sealer. Braz Oral Res 2006;20:303-6

[19]. Fraga RC, Siqueira JF Jr, de Uzeda M. In vitro evaluation of antibacterial effects of photo-cured glass ionomer liners and dentin bonding agents during setting. J Prosthet Dent. 1996;76:483-6.

[20]. Torabinejad M, Hong CU, Pitt Ford TR, Kettering JD. Antibacterial effects of some root end filling materials. J Endod 1995;21:403-6.

[21]. Stowe TJ, Sedgley CM, Stowe B, Fenno JC. The effects of chlorhexidine gluconate $(0.12 \%)$ on the antimicrobial properties of tooth-colored ProRoot mineral trioxide aggregate. J Endod 2004;30:429-31.

[22]. Yasuda Y, Kamaguchi A, Saito T. In vitro evaluation of the antimicrobial activity of a new resin-based endodontic sealer against endodontic pathogens. J Oral Sci 2008;50:309-13.

[23]. Al-Hezaimi K, Al-Shalan TA, Naghshbandi J, Oglesby S, Simon JH, Rotstein I. Antibacterial effect of two mineral trioxide aggregate (MTA) preparations against Enterococcus faecalis and Streptococcus sanguis in vitro. J Endod 2006;32:1053-6.

[24]. Zhang H, Shen Y, Ruse ND, Haapasalo M. Antibacterial activity of endodontic sealers by modified direct contact test against Enterococcus faecalis. J Endod 2009;35:1051-5.

[25]. Filho MT, Tanomaru JMG, Barros DB, Watanabe E, Ito IY. In vitro antimicrobial activity of endodontic sealers, MTA-based cements and Portland cement. J oral Scie 2007;49:41-5.

[26]. Tanomaru JMG, Filho MT, Hotta J, Watanabe E, Ito IY. Antimicrobial activity of endodontic sealers based on calcium hydroxide and MTA. Acta Odontol Latinoam 2008;21:147-51.

[27]. Pinheiro CR, Guinesi AS, Pizzolitto AC, Filho IB. In Vitro Antimicrobial Activity of Acroseal, Polifil and Epiphany against Enterococcus faecalis. Braz Dent J 2009; 20: 107-11.

[28]. Keshtkar T, Riaz F. The antibacterial activity of three endodontic sealers against Enterococcus faecalis in vitro. Master Thesis, December 5th, 2014; 1-10. 